\title{
The effects of direct hemoperfusion with polymyxin B-immobilized fiber in patients with acute exacerbation of interstitial lung disease
}

\author{
Jae Ha Lee ${ }^{1, *}$, Jin Han Park ${ }^{1, *}$, Hyo-Jung Kim¹, Hyun Kuk Kim ${ }^{1}$, Ji Hoon Jang', Yong Kyun Kim², \\ Bong Soo Park ${ }^{3}$, Si Hyung Park ${ }^{3}$, Il Hwan Kim ${ }^{4}$, Se Hun Kim ${ }^{5}$, Woon Heo ${ }^{6}$, Hang-Jea Jang ${ }^{1}$ \\ ${ }^{1}$ Division of Pulmonology, Department of Internal Medicine, Inje University Haeundae Paik Hospital, Inje University College of Medicine, Busan; \\ ${ }^{2}$ Division of Infectious Diseases, Department of Internal Medicine, Hallym University Sacred Heart Hospital, Hallym University College of Medicine, Anyang; \\ Divisions of ${ }^{3}$ Nephrology and ${ }^{4}$ Oncology, Department of Internal Medicine and Departments of ${ }^{5}$ Anesthesiology and ${ }^{6}$ Thoracic and Cardiovascular Surgery, \\ Inje University Haeundae Paik Hospital, Inje University College of Medicine, Busan, Korea
}

Background: Acute exacerbation of interstitial lung disease (AE-ILD) causes clinically significant deterioration and has an extremely poor prognosis with high mortality. Recently, several studies reported the effectiveness of direct hemoperfusion with a polymyxin B-immobilized fiber column (PMX-DHP) in patients with AE-ILD as a potential therapy. This study describes the clinical effectiveness and safety of PMX-DHP in patients with AE-ILD.

Methods: We retrospectively reviewed the medical records of 10 patients (11 episodes) with AE-ILD treated with PMX-DHP from January 2018 to June 2019. We compared laboratory and physiologic data of the ratio of partial pressure arterial oxygen to fraction of inspired oxygen ( $P / F$ ratio) and level of inflammatory markers before and after implementation of PMX-DHP. Results: Ten patients were included according to the 2016 revised definition of acute exacerbation of idiopathic pulmonary fibrosis (IPF). Nine patients had IPF and one patient had fibrotic nonspecific interstitial pneumonia. Most patients (90.9\%) were treated with a steroid pulse, and four patients (36.4\%) were treated with an immunosuppressant. The median number of PMX-DHP cycles was 2, and the median duration of each cycle was 6 hours. After PMXDHP, the mean $P / F$ ratio improved (86 [range, 63-106] vs. 145 [86-260], $P=0.030$ ) and interleukin-6 and c-reactive protein decreased (79 [35-640] vs. 10 [5-25], $P=0.018$ and 14 [4-21] vs. $5[2-6], P=0.019$, respectively). The 30 -day mortality rate was $27.3 \%$ and the 90 -day mortality rate was $72.7 \%$.

Conclusions: PMX-DHP treatment improved P/F ratio and reduced inflammatory markers in AE-ILD patients.

Key Words: acute exacerbation; idiopathic pulmonary fibrosis; interstitial lung disease; polymyxin B-immobilized fiber column

\section{INTRODUCTION}

Acute exacerbation of interstitial lung disease (AE-ILD) is a life-threatening event with very poor prognosis [1]. The mortality for idiopathic pulmonary fibrosis (IPF) is estimated at greater than $50 \%$, with a median survival of only 1-4 months [2-5]. Despite increasing recognition

\section{Original Article}

Received: January 13, 2021

Revised: March 3, 2021

Accepted: March 3, 2021

\section{Corresponding author}

Hang-Jea Jang

Department of Internal Medicine, Inje University Haeundae Paik Hospital, Inje University College of Medicine, 875 Haeun-daero, Haeundae-gu, Busan 48108, Korea Tel: +82-51-797-0100

Fax: +82-51-797-3009 E-mail: okabango21@gmail.com

*These authors contributed equally to this work.

Copyright () 2021 The Korean Society of Critical Care Medicine

This is an Open Access article distributed under the terms of Creative Attributions Non-Commercial License (https:// creativecommons.org/li-censes/by-nc/4.0/ which permits unrestricted noncommercial use, distribution, and reproduction in any medium, provided the original work is properly cited. 
and intervention efforts for AE-ILD, no treatment has demonstrated clinical benefit. Corticosteroids and immunosuppressive drugs have been used to treat AE-ILD patients, but new therapies with high efficacy are needed.

Polymyxin B-immobilized fiber column (PMX-DHP) was originally developed to adsorb endotoxins released by Gramnegative bacteria during septic shock [6]. Other studies have found that PMX-DHP might be useful for patients with acute respiratory distress syndrome and acute lung injury, which are pathologically characterized by diffuse alveolar damage $[7,8]$. The actual mechanism of PMX-DHP in AE-ILD patients is not understood well. However, an in vitro study recently suggested that absorption of various cytokines involved in inflammation, fibrosis, and vascular permeability might be associated with the positive impacts of PMX-DHP treatments [9]. Several recent reports have shown that PMX-DHP treatment has beneficial effects on oxygenation, inflammatorymarker removal, and long-term outcomes in AE-ILD patients [10-12]. However, the clinical outcomes and survival benefits have not been accurately evaluated. In addition, most previous studies were performed in Japan. Therefore, the aim of this study was to evaluate the clinical effects and safety of PMXDHP in Korean patients with AE-ILD.

\section{MATERIALS AND METHODS}

This study was approved by the Institutional Review Board of Haeundae Paik Hospital (IRB No. 2019-08-021), and the requirement for written informed consent was waived due to the retrospective nature.

\section{Study Subjects}

Clinical data from 10 AE-ILD patients (11 AE episodes) who were treated with PMX-DHP from January 2018 to June 2019 at Haeundae Paik Hospital, Busan, Republic of Korea, were retrospectively analyzed. Diagnosis of ILD was based on international guidelines $[13,14]$. The diagnostic criteria for AEILD based on Collard et al. [15] were as follows: (1) previous or concurrent IPF diagnosis; (2) acute worsening or development of dyspnea typically within the past month; (3) high-resolution computed tomography (HRCT) with new bilateral ground-glass opacity and/or consolidation superimposed on a background pattern consistent with a typical interstitial pneumonia pattern; and (4) deterioration not fully explained by cardiac failure or fluid overload [15].

\section{KEY MESSAGES}

- Polymyxin B-immobilized fiber column (PMX-DHP) treatment for acute exacerbation of interstitial lung disease (AE-ILD) patients improved the ratio of partial pressure arterial oxygen to fraction of inspired oxygen and decreased inflammatory markers, which might improve clinical outcomes.

- Further studies including a double-blind randomized controlled study are needed to confirm the clinical effects and survival benefit of PMX-DHP in AE-ILD patients.

\section{Clinical Information}

Baseline characteristics and survival data were obtained from medical records. Spirometry, diffusing capacity of the lung for carbon monoxide (DLCO), and forced vital capacity (FVC) were determined according to recommendations from the American Thoracic Society (ATS) and the European Respiratory Society and are reported as percentage of normal predicted value $[16,17]$. The 6 -minute walk test (6MWT) was performed according to ATS guidelines [18]. IPF severity was classified according to gender-age-physiology (GAP) models, which were used to predict IPF progression [19].

\section{PMX-DHP Treatment}

After AE-ILD diagnosis, patients were treated with a corticosteroid pulse (500 mg or $1 \mathrm{~g}$ of methylprednisolone over 1 day) and antibiotics as conventional therapy. Simultaneously, PMXDHP treatment was started after diagnosis in patients with severe AE-ILD. PMX-DHP treatment was performed for 6 hours per day over two consecutive days using a PMX column (Toraymyxin 20R; Toray Medical, Tokyo, Japan). Before use, a PMXfiber cartridge was rinsed with $4 \mathrm{~L}$ physiological saline and primed with 2,000 $\mathrm{U}$ heparin sodium dissolved in $0.5 \mathrm{~L}$ saline. Blood access for direct hemoperfusion with PMX-DHP was obtained using a double-lumen catheter inserted into the jugular or femoral vein. The PMX-fiber cartridge was connected to a polymethyl methacrylate membrane (Hemofeel $\mathrm{CH}-1.0 \mathrm{~N}$, Toray Medical) that returned blood to the femoral vein. The hemoperfusion flow rate was $120-150 \mathrm{~mL} / \mathrm{min}$. Nafamostat mesilate (NM; Toril Pharmaceuticals, Tokyo, Japan) was administered into a continuous renal replacement therapy circuit as a regional anticoagulant.

\section{Assessment of PMX-DHP Effects}

Serum inflammatory markers of white blood cells (WBCs), c- 
reactive protein (CRP), interleukin (IL)-6, and the ratio of partial pressure arterial oxygen to fraction of inspired oxygen (P/ F ratio) were measured before and 48 hours after PMX-DHP treatment. IL-6 level was measured by electrochemiluminescence immunoassay using the Cobas e411 analyzer (Hitachi High-Technologies, Tokyo, Japan). We compared the results before and 48 hours after PMX-DHP treatment.

\section{Statistical Analysis}

Data are presented as frequency with percentage for categorical variables and as median and interquartile range for continuous variables. Paired t-test or Wilcoxon's signed-rank test was performed for comparison between two assessment time points. To determine normal distribution, we used the Shapiro-Wilk test. The generalized linear mixed model was employed for longitudinal data to adjust covariates. Cox's multivariate regression models were fit to identify prognostic factors independently related to overall survival, which was estimated using the Kaplan-Meier curve with spaghetti plots for data visualization. All statistical analyses were performed using IBM SPSS ver. 24.0 (IBM Corp., Armonk, NY, USA), and Pvalues $<0.05$ were considered statistically significant.

\section{RESULTS}

\section{Baseline Clinical Characteristics}

Ten patients (11 episodes) were included in this study. Nine patients (10 episodes) were clinically diagnosed with IPF and one patient was diagnosed with fibrotic nonspecific interstitial pneumonia by surgical lung biopsy. The median patient age was 66 years (range, 62-74 years), and seven patients (63.6\%) were male. The median duration from diagnosis of ILD to acute exacerbation of ILD was 13 months (range, 3-32 months). Three patients (27.3\%) had a history of lung cancer. Most patients showed moderately restrictive lung function (median FVC, $51 \%$ of predicted; range, $47 \%-66 \%$ ) and low DLCO (median, $37 \%$ of predicted; range, $35 \%-46 \%$ ) on pulmonary function testing. Functional capacity (total distance and de-saturation during 6MWT) was poor. According to GAP stage, IPF severity was classified as stage I, II, or III, with stage II being most common (54.5\%). The median baseline Sequential Organ Failure Assessment (SOFA) score was 5 (range, 4-6), and six patients (54.5\%) suffered shock during their initial intensive care unit admission. At AE-ILD diagnosis, the median initial $\mathrm{P} / \mathrm{F}$ ratio was 86 (range, 64-107), representing severe hypoxemia, and $90 \%$ of patients received steroid pulse therapy, while $36.4 \%$ were concomitantly treated with immunosuppressants. Dur-
Table 1. Characteristic of patients treated with PMX-DHP

\begin{tabular}{|c|c|}
\hline Characteristics & Value \\
\hline Age (yr) & $66(62-74)$ \\
\hline Male sex & 7 (63.6) \\
\hline BMI $\left(\mathrm{kg} / \mathrm{m}^{2}\right)$ & $24.1(20.0-24.8)$ \\
\hline Lung cancer & $3(27.3)$ \\
\hline Hypertension & $2(18.2)$ \\
\hline Diabetes mellitus & $1(9.1)$ \\
\hline Period from ILD diagnosis to AE (mo) & $13(3-32)$ \\
\hline GAP stage (I:II:III) & $2: 6: 3$ \\
\hline SOFA score & $5(4-6)$ \\
\hline \multicolumn{2}{|l|}{ Pulmonary function } \\
\hline $\mathrm{FVC}, \%$ predicted & $51(47-66)$ \\
\hline FVC, L measured & $2.1(1.6-2.5)$ \\
\hline DLCO, \% predicted & $37(35-46)$ \\
\hline DLCO, L measured & $7.2(5.7-8.3)$ \\
\hline 6-Minute walk test & $11(100.0)$ \\
\hline Distance (m) & 345 (209-390) \\
\hline Initial $\mathrm{SpO}_{2}(\%)$ & 97 (90-98) \\
\hline Lowest $\mathrm{SpO}_{2}(\%)$ & $87(76-93)$ \\
\hline Initial $\mathrm{P} / \mathrm{F}$ ratio & $86(64-107)$ \\
\hline $\mathrm{PaO}_{2}(\mathrm{~mm} \mathrm{Hg})$ & $64(52-71)$ \\
\hline $\mathrm{PaCO}_{2}(\mathrm{~mm} \mathrm{Hg})$ & $34(33-40)$ \\
\hline Serum WBC $\left(\times 10^{6} / \mathrm{L}\right)$ & $16,260(14,400-22,210)$ \\
\hline Serum CRP (mg/dl) & $14.9(3.7-20.7)$ \\
\hline Serum LDH (U/L) & $466(428-582)$ \\
\hline Serum lactate (mmol/L) & $2.0(1.5-3.0)$ \\
\hline Shock $^{\mathrm{a}}$ & $6(54.5)$ \\
\hline Mechanical ventilation & 7 (63.6) \\
\hline Steroid pulse therapy & 10 (90.9) \\
\hline 500 mg methylPD per day & $5(45.5)$ \\
\hline $1 \mathrm{~g}$ methylPD per day & $5(45.5)$ \\
\hline Immunosuppressant & $4(36.4)$ \\
\hline Cyclosporin & $4(36.4)$ \\
\hline Cyclophosphamide & $2(18.2)$ \\
\hline ECMO & $4(36.4)$ \\
\hline 30-Day mortality & $3(27.3)$ \\
\hline 90-Day mortality & $8(72.7)$ \\
\hline
\end{tabular}

Values are presented as median (interquartile range) or number (\%). PMX-DHP: polymyxin B-immobilized fiber column; BMI: body mass index; ILD: interstitial lung disease; AE: acute exacerbation; GAP stage: genderage-physiology stage; SOFA: Sequential Organ Failure Assessment; FVC: forced vital capacity; DLCO: diffusing capacity of the lung for carbon monoxide; $\mathrm{SpO}_{2}$ : saturation of peripheral oxygen; P/F ratio: the ratio of partial pressure arterial oxygen to fraction of inspired oxygen; WBC: white blood cell; CRP: C-reactive protein; LDH: lactate dehydrogenase; methylPD: methylprednisolone; ECMO: extracorporeal membrane oxygenation. aShock: use of inotropic agent or vasopressor to maintain adequate tissue perfusion (mean arterial pressure greater than $65 \mathrm{~mm} \mathrm{Hg}$ ). 
Table 2. Changes in P/F ratio, IL-6, WBC, and CRP after 48 hours of PMX-DHP treatment

\begin{tabular}{lcccc}
\hline Variable & Before 1st session & 48 Hours later & Crude P-value $^{\text {Adjusted P-value }}$ \\
\hline P/F ratio & $86(63-106)$ & $145(86-260)$ & 0.030 & 0.035 \\
IL-6 $(\mathrm{pg} / \mathrm{ml})$ & $79(35-640)$ & $10(5-25)$ & 0.018 & 0.408 \\
WBC $\left(\times 10^{6} / \mathrm{L}\right)$ & $16,260(14,400-22,210)$ & $14,070(9,132-23,917)$ & 0.386 & 0.608 \\
CRP $(\mathrm{mg} / \mathrm{dl})$ & $14(4-21)$ & $5(2-6)$ & 0.019 & 0.024 \\
\hline
\end{tabular}

Values are presented as median (interquartile range).

P/F ratio: the ratio of partial pressure arterial oxygen to fraction of inspired oxygen; IL: interleukin; WBC: white blood cell; CRP: C-reactive protein; PMX-DHP: polymyxin B-immobilized fiber column.

${ }^{a}$ P-values were adjusted for application of mechanical ventilation, use of steroid pulse therapy, steroid dose, and use of immunosuppressant.
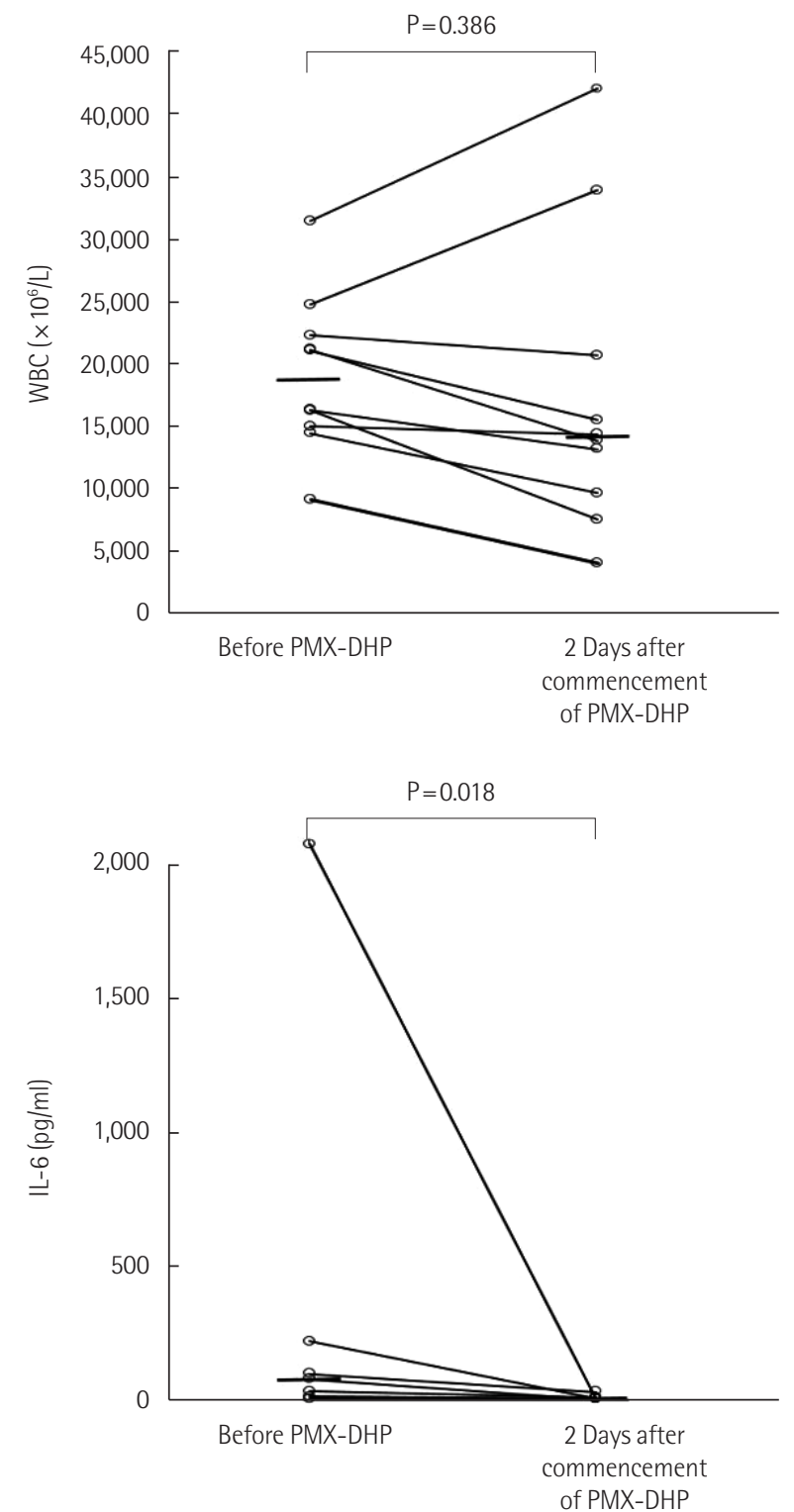

A
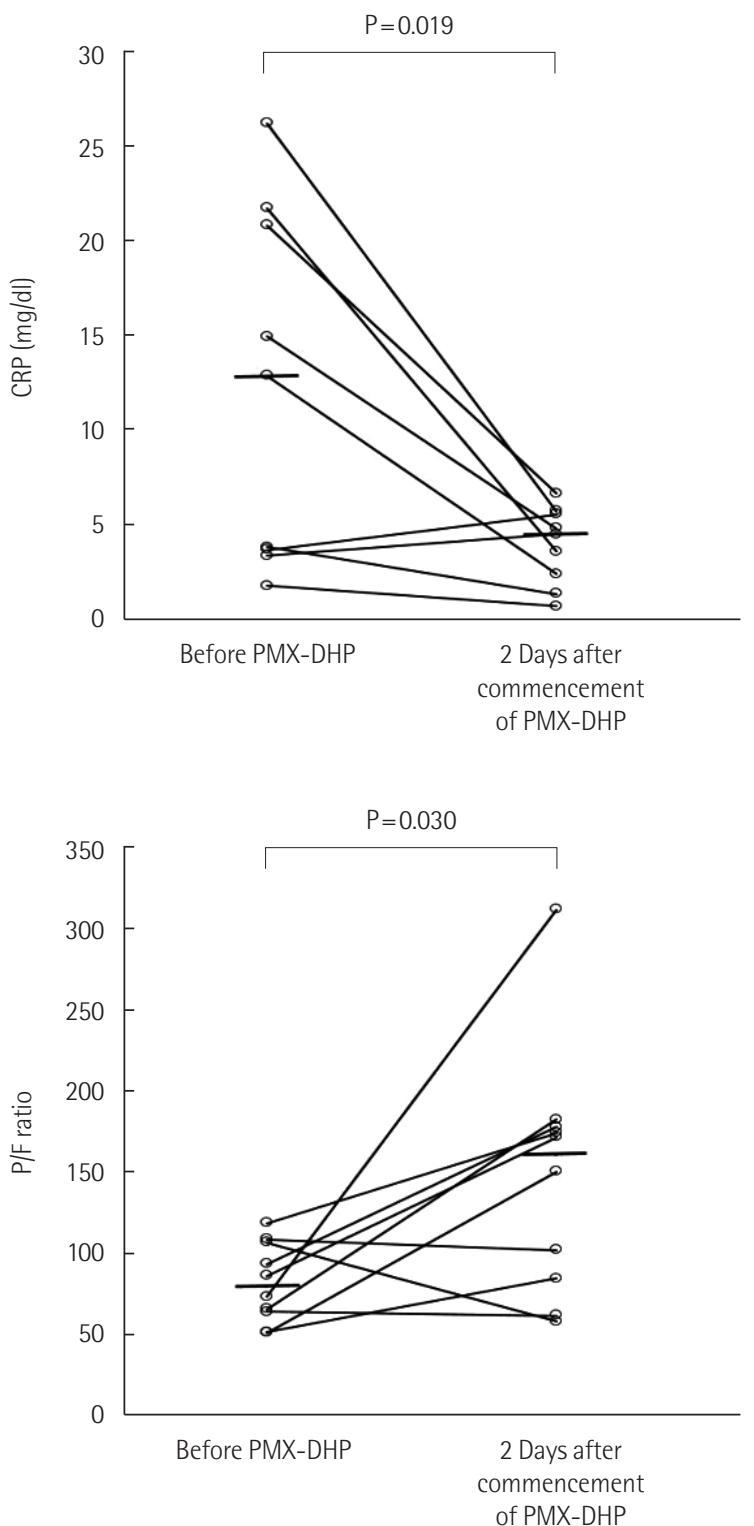

B

(D)

Figure 1. Comparison of white blood cell (WBC), C-reactive protein (CRP), interleukin (IL)-6, and the ratio of partial pressure arterial oxygen to fraction of inspired oxygen (P/F ratio) at 48 hours after polymyxin B-immobilized fiber column (PMX-DHP) treatment. (A) WBC count, (B) CRP, (C) IL-6, (D) P/F ratio. Serum levels of CRP and IL-6 were decreased after 48 hours of PMX-DHP. The P/F ratio was improved after 48 hours of PMX-DHP. There was no change in WBC count at after 48 hours of PMX-DHP. The horizontal bars indicate median values. 


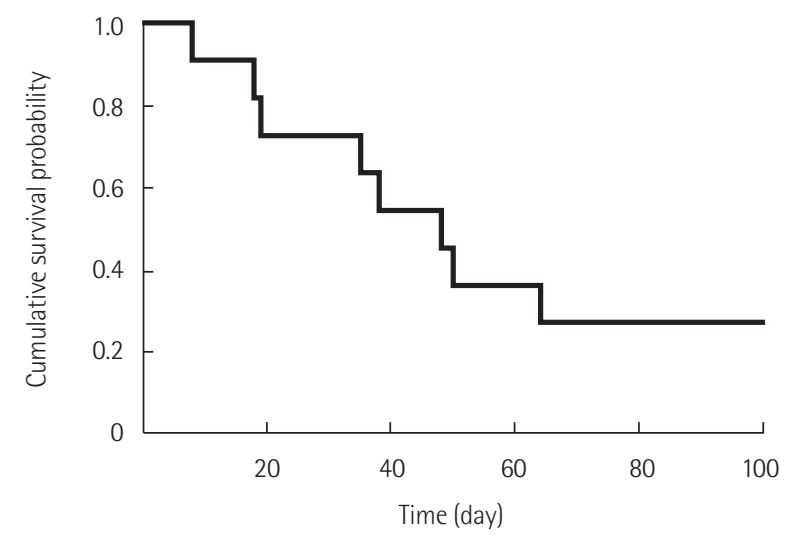

Figure 2. Kaplan-Meier survival curve for patients with acute exacerbation of interstitial lung disease (AE-ILD) treated with polymyxin B-immobilized fiber column (PMX-DHP). In patients with AE-ILD treated by PMX-DHP, the 30-day mortality rate was $27.3 \%$ and the 90 -day mortality rate was $72.7 \%$.

ing treatment with PMX-DHP, six patients (seven episodes) required mechanical ventilator support, and four patients were supported by high-flow nasal cannula (HFNC). After treatment with PMX-DHP, four patients (36.4\%) were treated with venovenous extracorporeal membrane oxygenation (ECMO) therapy, one of whom survived (Table 1).

\section{Details and Effects of PMX-DHP}

The median time from AE-IPF onset to PMX-DHP application was 2 days (range, 0-3 days). The median number of PMXDHP cycles was 2 (range, 2-3), and the median cycle duration was 6 hours (range, 6-8 hours). The median total duration of PMX-DHP treatment was 12.0 hours (range, 12-24 hours). NM was used as an anticoagulant in all patients, and there were no complications associated with hemoperfusion. At 48 hours after treatment, IL-6 and CRP decreased (Table 2), change in WBC was not significant, and the $\mathrm{P} / \mathrm{F}$ ratio was improved significantly (86 [range, 63-106] vs. 145 [range, 86-260], $\mathrm{P}=0.030$ ) compared to pretreatment values (Figure 1). However, after adjusting for mechanical ventilation, use of steroid pulse therapy, steroid dose, and use of immunosuppressant, improvement of $\mathrm{P} / \mathrm{F}$ ratio and decrease of CRP remained significant, but change in IL-6 did not.

\section{Survival Analysis}

During the follow-up period, the survival rate from the time of AE onset using Kaplan-Meier analysis was $72.7 \%$ at 30 days and $27.3 \%$ at 90 days (Figure 2). The median survival period was 48 days (range, $8-570$ days). Among four patients who received ECMO, 1 (25\%) was weaned from treatment and sur- vived for 570 days. The most common cause of death was acute respiratory failure including pneumonia $(57.1 \%)$, followed by multi-organ failure (28.6\%) and cardiovascular events (14.3\%).

\section{DISCUSSION}

In our study, the clinical effects of PMX-DHP in AE-ILD patients were similar to those reported previously. PMX-DHP improved the $\mathrm{P} / \mathrm{F}$ ratio and removal of inflammatory markers compared with pre-PMX-DHP treatment levels. Despite clinical improvement including $\mathrm{P} / \mathrm{F}$ ratio and removal of inflammatory markers, mortality was high and clinical improvement did not result in survival for most patients. There were no adverse events associated with PMX-DHP treatment.

Several previous studies reported that PMX-DHP treatment reduces many kinds of inflammatory markers [6,20,21]. Oishi et al. [22] reported that, in nine AE-IPF patients who were treated with PMX-DHP, serum CRP, IL-9 (median, $35.6 \mathrm{pg} / \mathrm{ml}$ vs. $28.2 \mathrm{pg} / \mathrm{ml} ; \mathrm{P}=0.011$ ), and IL-12 (median, $38.8 \mathrm{pg} / \mathrm{ml}$ vs. 12.6 $\mathrm{pg} / \mathrm{ml} ; \mathrm{P}=0.004)$ significantly decreased after treatment. In a study including 14 patients (20 episodes) treated with PMXDHP, Enomoto et al. [23] reported a significant change in neutrophil count $(1,095 \pm 1,649$ vs. $6,166 \pm 1,406, P=0.031)$ and angiopoietin-2 before and after PMX-DHP. Our study also confirmed the effectiveness of PMX-DHP as an inflammatorymarker reducer, where we found significant differences in IL-6 and CRP but not in WBC. Among many inflammatory markers or cytokines, we examined CRP and serum IL- 6 because IL-6 was previously established as a useful biomarker for AE-ILD given its roles as a soluble mediator with pleiotropic effects on inflammation, immune response, and fibrosis [24]. Papiris et al. [25] compared cytokine levels between 23 stable IPF and 19 AE-IPF patients and reported that high IL-6 level is characteristic of AE-IPF, and that increased IL-6 is associated with worse outcomes.

Previous studies found that PMX-DHP treatment improved the $\mathrm{P} / \mathrm{F}$ ratio in AE-ILD patients. Our study also showed that PMX-DHP treatment had a tendency to improve the $\mathrm{P} / \mathrm{F}$ ratio. A previous study of five AE-IPF patients who received PMXDHP corroborates our findings of improved P/F ratio (170.0 \pm 119.4 vs. $217.1 \pm 129.6, \mathrm{P}=0.02$ ) [26]. The actual mechanism of oxygenation improvement by PMX-DHP is not understood fully, but Abe et al. [27] suggested that active and inflammatory neutrophils that are absorbed by the PMX column play a role.

Our 30-day mortality rate was $27.3 \%$ and the 90 -day mortality rate was $72.7 \%$, which is higher than in other reports $[28,29]$. In our study, the pre-PMX-DHP treatment $\mathrm{P} / \mathrm{F}$ ratio was very 
low (median, 86; range, 63-106), suggesting that our patient population was critically ill despite rapid application of PMXDHP (median time from AE-IPF onset to PMX-DHP: 2 days). In addition, all patients were treated for severe respiratory failure with mechanical ventilation and HFNC in the intensive care unit. We assume that the disease severity of our enrolled AE-ILD patients might be the reason for our high mortality rate. In addition, clinical improvement did not result in longterm survival in most patients. This could be because shortterm clinical improvement was not enough to avoid continuous mechanical ventilation or ECMO care, which is critical to survival. However, the effect of clinical improvement from PMX-DHP on survival benefit was uncertain in this study. As a pilot study, this will be helpful for additional large-scale and well-designed studies in the near future in Korea.

This study has some limitations. First, this was a small retrospective study at a single center. However, patients were treated with almost the same protocol, including PMX-DHP and conventional AE-ILD treatment by a single pulmonologist. All patients were treated with PMX-DHP within 4 days of AE-ILD onset. This study was composed of data gathered within a twoyear period, reducing bias from varied medical conditions over time. Second, there was no control group (without PMXDHP treatment), so it was difficult to accurately assess the effect of PMX-DHP treatment. Third, we could not examine all inflammatory markers and cytokines that could be important. It is possible that other inflammatory markers and cytokines are relevant to AE-ILD pathogenesis.

In conclusion, PMX-DHP treatment for AE-ILD patients showed improvement in the $\mathrm{P} / \mathrm{F}$ ratio and a decrease in inflammatory markers. For some patients, these improvements in clinical aspects might lead to better clinical outcomes. To confirm the clinical effects and survival benefits of PMX-DHP in AE-ILD patients, more studies are needed, including a double-blind, randomized, controlled study.

\section{CONFLICT OF INTEREST}

No potential conflict of interest relevant to this article was reported.

\section{ORCID}

Jae Ha Lee

Jin Han Park

https://orcid.org/0000-0003-0932-2826

Hyo-Jung Kim https://orcid.org/0000-0002-1138-4957

Hyun Kuk Kim
Ji Hoon Jang

Yong Kyun Kim

Bong Soo Park

Si Hyung Park

Il Hwan Kim

Se Hun Kim

Woon Heo

Hang-Jea Jang https://orcid.org/0000-0002-5048-8820 https://orcid.org/0000-0002-7935-6952 https://orcid.org/0000-0001-8999-386X https://orcid.org/0000-0002-6782-5299 https://orcid.org/0000-0003-4166-6303 https://orcid.org/0000-0002-2752-2883 https://orcid.org/0000-0001-8208-484X https://orcid.org/0000-0001-7733-4365

\section{AUTHOR CONTRIBUTIONS}

Conceptualization: JHL, HJJ. Data curation: JHL, JHP, JHJ, SHK, WH. Formal analysis \& Methodology: JHL, JHP, HJK, HKK, YKK, IHK. Visualization: JHL, JHP, BSP, SHP. Writingoriginal draft: JHL, JHP. Writing-review \& editing: all authors.

\section{REFERENCES}

1. Leuschner G, Behr J. Acute exacerbation in interstitial lung disease. Front Med (Lausanne) 2017;4:176.

2. Usui Y, Kaga A, Sakai F, Shiono A, Komiyama K, Hagiwara K, et al. A cohort study of mortality predictors in patients with acute exacerbation of chronic fibrosing interstitial pneumonia. BMJ Open 2013;3:e002971.

3. Song JW, Hong SB, Lim CM, Koh Y, Kim DS. Acute exacerbation of idiopathic pulmonary fibrosis: incidence, risk factors and outcome. Eur Respir J 2011;37:356-63.

4. Kim DS, Park JH, Park BK, Lee JS, Nicholson AG, Colby T. Acute exacerbation of idiopathic pulmonary fibrosis: frequency and clinical features. Eur Respir J 2006;27:143-50.

5. Al-Hameed FM, Sharma S. Outcome of patients admitted to the intensive care unit for acute exacerbation of idiopathic pulmonary fibrosis. Can Respir J 2004;11:117-22.

6. Shoji H. Extracorporeal endotoxin removal for the treatment of sepsis: endotoxin adsorption cartridge (Toraymyxin). Ther Apher Dial 2003;7:108-14.

7. Nakamura T, Kawagoe Y, Matsuda T, Shoji H, Ueda Y, Tamura $\mathrm{N}$, et al. Effect of polymyxin B-immobilized fiber on blood metalloproteinase-9 and tissue inhibitor of metalloproteinase- 1 levels in acute respiratory distress syndrome patients. Blood Purif 2004;22:256-60.

8. Tsushima K, Kubo K, Koizumi T, Yamamoto H, Fujimoto K, Hora K, et al. Direct hemoperfusion using a polymyxin B immobilized column improves acute respiratory distress syndrome. J Clin Apher 2002;17:97-102.

9. Utsunomiya T, Mimura-Kimura Y, Yamamoto T, Aoe K, Oishi $\mathrm{K}$, Kamei H, et al. Cytokine adsorption to polymyxin B-immo- 
bilized fiber: an in vitro Study. Blood Purif 2021;50:230-7.

10. Abe S, Azuma A, Mukae H, Ogura T, Taniguchi H, Bando M, et al. Polymyxin B-immobilized fiber column (PMX) treatment for idiopathic pulmonary fibrosis with acute exacerbation: a multicenter retrospective analysis. Intern Med 2012;51:148791.

11. Hara S, Ishimoto H, Sakamoto N, Mukae H, Kakugawa T, Ishimatsu Y, et al. Direct hemoperfusion using immobilized polymyxin B in patients with rapidly progressive interstitial pneumonias: a retrospective study. Respiration 2011;81:107-17.

12. Seo Y, Abe S, Kurahara M, Okada D, Saito Y, Usuki J, et al. Beneficial effect of polymyxin B-immobilized fiber column (PMX) hemoperfusion treatment on acute exacerbation of idiopathic pulmonary fibrosis. Intern Med 2006;45:1033-8.

13. Travis WD, Costabel U, Hansell DM, King TE Jr, Lynch DA, Nicholson AG, et al. An official American Thoracic Society/ European Respiratory Society statement: update of the international multidisciplinary classification of the idiopathic interstitial pneumonias. Am J Respir Crit Care Med 2013;188: 733-48.

14. Raghu G, Collard HR, Egan JJ, Martinez FJ, Behr J, Brown KK, et al. An official ATS/ERS/JRS/ALAT statement: idiopathic pulmonary fibrosis: evidence-based guidelines for diagnosis and management. Am J Respir Crit Care Med 2011;183:788824.

15. Collard HR, Ryerson CJ, Corte TJ, Jenkins G, Kondoh Y, Lederer DJ, et al. Acute exacerbation of idiopathic pulmonary fibrosis. an international working group report. Am J Respir Crit Care Med 2016;194:265-75.

16. Wanger J, Clausen JL, Coates A, Pedersen OF, Brusasco V, Burgos $\mathrm{F}$, et al. Standardisation of the measurement of lung volumes. Eur Respir J 2005;26:511-22.

17. Miller MR, Hankinson J, Brusasco V, Burgos F, Casaburi R, Coates A, et al. Standardisation of spirometry. Eur Respir J 2005; 26:319-38.

18. Holland AE, Spruit MA, Troosters T, Puhan MA, Pepin V, Saey D, et al. An official European Respiratory Society/American Thoracic Society technical standard: field walking tests in chronic respiratory disease. Eur Respir J 2014;44:1428-46.

19. Ley B, Ryerson CJ, Vittinghoff E, Ryu JH, Tomassetti S, Lee JS, et al. A multidimensional index and staging system for idiopathic pulmonary fibrosis. Ann Intern Med 2012;156:684-91.

20. Ueno T, Ikeda T, Ikeda K, Taniuchi H, Suda S, Yeung MY, et al.
HMGB-1 as a useful prognostic biomarker in sepsis-induced organ failure in patients undergoing PMX-DHP. J Surg Res 2011;171:183-90.

21. Tani T, Hanasawa K, Kodama M, Imaizumi H, Yonekawa M, Saito M, et al. Correlation between plasma endotoxin, plasma cytokines, and plasminogen activator inhibitor-1 activities in septic patients. World J Surg 2001;25:660-8.

22. Oishi K, Mimura-Kimura Y, Miyasho T, Aoe K, Ogata Y, Katayama $\mathrm{H}$, et al. Association between cytokine removal by polymyxin B hemoperfusion and improved pulmonary oxygenation in patients with acute exacerbation of idiopathic pulmonary fibrosis. Cytokine 2013;61:84-9.

23. Enomoto N, Mikamo M, Oyama Y, Kono M, Hashimoto D, Fujisawa T, et al. Treatment of acute exacerbation of idiopathic pulmonary fibrosis with direct hemoperfusion using a polymyxin B-immobilized fiber column improves survival. BMC Pulm Med 2015;15:15.

24. Tanaka T, Narazaki M, Kishimoto T. IL-6 in inflammation, immunity, and disease. Cold Spring Harb Perspect Biol 2014;6: a016295.

25. Papiris SA, Tomos IP, Karakatsani A, Spathis A, Korbila I, Analitis A, et al. High levels of IL-6 and IL-8 characterize early-on idiopathic pulmonary fibrosis acute exacerbations. Cytokine 2018;102:168-72

26. Kono M, Suda T, Enomoto N, Nakamura Y, Kaida Y, Hashimoto D, et al. Evaluation of different perfusion durations in direct hemoperfusion with polymyxin B-immobilized fiber column therapy for acute exacerbation of interstitial pneumonias. Blood Purif 2011;32:75-81.

27. Abe S, Seo Y, Hayashi H, Matsuda K, Usuki J, Azuma A, et al. Neutrophil adsorption by polymyxin B-immobilized fiber column for acute exacerbation in patients with interstitial pneumonia: a pilot study. Blood Purif 2010;29:321-6.

28. Takada T, Asakawa K, Sakagami T, Moriyama H, Kazama J, Suzuki E, et al. Effects of direct hemoperfusion with polymyxin B-immobilized fiber on rapidly progressive interstitial lung diseases. Intern Med 2014;53:1921-6.

29. Ichiyasu H, Horio Y, Masunaga A, Migiyama Y, Sakamoto Y, Jodai T, et al. Efficacy of direct hemoperfusion using polymyxin B-immobilized fiber column (PMX-DHP) in rapidly progressive interstitial pneumonias: results of a historical control study and a review of previous studies. Ther Adv Respir Dis 2017;11:261-75. 